\title{
Arthroscopic Release of Resistant Tennis Elbow
}

Faisal Hassan Zayed ${ }^{(1)}$ Ismael Ahmed Yassin ${ }^{(1)}$ Waleed Abd-Allah Salama Al-Attar ${ }^{(2)}$

Department of Orthopedic Surgery, Al-Azhar Faculty of Medicine ${ }^{(1)}$,

Department of Orthopedic Surgery, Al-Azhar university hospital, Cairo ${ }^{(2)}$

Corresponding Author: Waleed Abd-Allah Salama Al-Attar, Mob: 01090745436 E-mail: waleedelattar4@gmail.com

\begin{abstract}
Background: Lateral epicondylitis is a common cause of lateral elbow pain; characterized as an overuse injury of the forearm and the wrist extensors. The elbow arthroscopy has an important role for diagnosing and treating many elbow disorders. Objective: The aim of this study was to evaluate the effectiveness of the arthroscopic intervention as a minimally invasive procedure in management of resistant cases with tennis elbow after failure of conservative treatment. Material and methods: A prospective study included 30 patients with resistant lateral epicondylitis treated with arthroscopic debridement of extensor carpi radialis brevis tendon. This study was carried out in the period between January 2017 and February 2019 at Al Azhar University Hospitals. The patients were evaluated pre-, intraand post-operatively for their elbow function and pain using the following scores: Mayo Elbow Performance Index (MEPI), Disability of the Arm, Shoulder and Hand (DASH), The Nirschl staging score and visual analog scale (VAS) for pain. Result: A total of 30 patients (12 male and 18 female) were included. The mean of MEPI score improved from 71.33 to 92.55 , DASH score from 28.53 to 3.18 , Nirschl scale improved from 4.13 to 1.47 and pain VAS improved from 5.27 to 1.47 . There was a significant difference between from before to after surgery for the four functional scores. 2 patients complicated with ulnar nerve injury.

Conclusion: The arthroscopic treatment of tennis elbow was shown to be an effective therapeutic option when appropriately indicated and performed in refractory cases. It also allowed excellent viewing of joint space for diagnosis and treatment of any associated pathological conditions with a minimally invasive procedure. Keywords: lateral epicondylitis, elbow pain, arthroscopic release.
\end{abstract}

\section{NTRODUCTION}

Lateral epicondylitis occurs as a result of repetitive motions involving the forearm and elbow, such as the serving, forehand and backhand motions in tennis and other racquet sports. It is a common sports injury, occurring in up to $50 \%$ of athletes whose sports involve frequent overarm motions ${ }^{(1)}$.

However, the name "tennis elbow" can be misleading, because most people who develop it do not play tennis. The condition can affect anyone who engages in the repetitive motion of one or both forearms, either in sports or during daily activities. The non-inflammatory nature of tendinopathy has been stressed $^{(2,3)}$ and it has become clear that tennis elbow does not involve an inflammatory process of the common extensor origin. It was proposed that the pathology is angiofibroblastic hyperplasia of the common extensor origin, especially of the tendon of the extensor capiradialis brevis (ECRB) ${ }^{(4)}$ in line with the other overuse tendinopathies ${ }^{(5)}$.

The diagnosis is mainly clinical, with image modalities such as ultrasound and MRI, to be used only for confirmatory, not diagnostic, purposes ${ }^{(6)}$.

The literature is vast regarding types of treatment, from rest to surgical intervention. However, controversy still exists regarding the best option of treatment. The initial management of lateral epicondylitis is conservative, with the use of rest, lifestyle modification, non-steroidal anti-inflammatory drugs, forearm bracing, ${ }^{(7)}$ physiotherapy and local steroid injection ${ }^{(8)}$. These measures result in at least a transient improvement in up to $90 \%$ of patients, and 3$8 \%$ of patients who are resistant to conservative treatment, may be surgical candidates ${ }^{(9)}$.
Conservative treatment presents with excellent results, although surgical treatment becomes an option when it fails (3-6 months) and in refractory chronic cases (approximately 12\%). The Arthroscopic treatment has recently been described as having the advantages of allowing viewing of the associated intra-articular lesions, not violating the aponeurosis of the extensors and having shorter rehabilitation period and lower complications rate ${ }^{(10)}$. In this study, 30 patients with resistant tennis elbow underwent an arthroscopic management and evaluated pre-, intra- and postoperatively for elbow function and pain using the following scores.

1) Mayo Elbow Performance Index (MEPI).

2) Disability of the Arm, Shoulder, Hand (DASH).

3) The Nirschl staging score.

4) Visual analogue scale (VAS) for pain ${ }^{(11)}$.

\section{PATIENTS AND METHODS}

From January 2017 to February 2019, 30 patients with resistant tennis elbow were treated with elbow arthroscopy at Orthopedic Department at AlAzhar University Hospitals (Bab El Sharia Hospitals). The follow up schedule occurred immediately, 2 weeks, 6 weeks and 6 months after the operation.

Inclusion criteria: Resistant cases after the failure of conservative treatments over a period of more than 6 months. Exclusion criteria: Presence of concomitant disorders, such as lateral compartment, arthrosis, posterior interosseous syndrome, osteochondritis dissecans of the capitellum and Instability and previous surgery or fracture on the ipsilateral elbow. 
Table (1): Demographic data in the studied patients.

\begin{tabular}{|c|c|c|c|}
\hline & & \multicolumn{2}{|c|}{ Patients } \\
\hline & & No. (30) & $\%$ \\
\hline \multirow[t]{2}{*}{$\overline{\text { Sex }}$} & Male & 12 & 40 \\
\hline & Female & 18 & 60 \\
\hline \multirow{2}{*}{ Age (years) } & $\overline{\text { Range }}$ & \multicolumn{2}{|c|}{$37-54$} \\
\hline & Mean \pm SD & \multicolumn{2}{|c|}{$44.2 \pm 5.96$} \\
\hline \multirow{2}{*}{ Dominant side } & Right & 24 & 80 \\
\hline & Left & 6 & 20 \\
\hline \multirow{2}{*}{ Affected side } & Right & 22 & 73.3 \\
\hline & Left & 8 & 26.7 \\
\hline \multirow{2}{*}{ Number of injections } & Range & \multicolumn{2}{|c|}{$2-5$} \\
\hline & Mean \pm SD & \multicolumn{2}{|c|}{$2.83 \pm 0.874$} \\
\hline \multirow{4}{*}{ Occupation } & House wife & 10 & 33.3 \\
\hline & Heavy worker & 16 & 53.3 \\
\hline & Nurse & 2 & 6.6 \\
\hline & Accountant & 2 & 6.6 \\
\hline
\end{tabular}

\section{Technique:}

All elbow arthroscopies were done in the lateral decubitus position. After the landmarks have been identified and marked on the skin, the limb was exsanguinated, and the tourniquet was inflated to $250 \mathrm{mmHg}$.

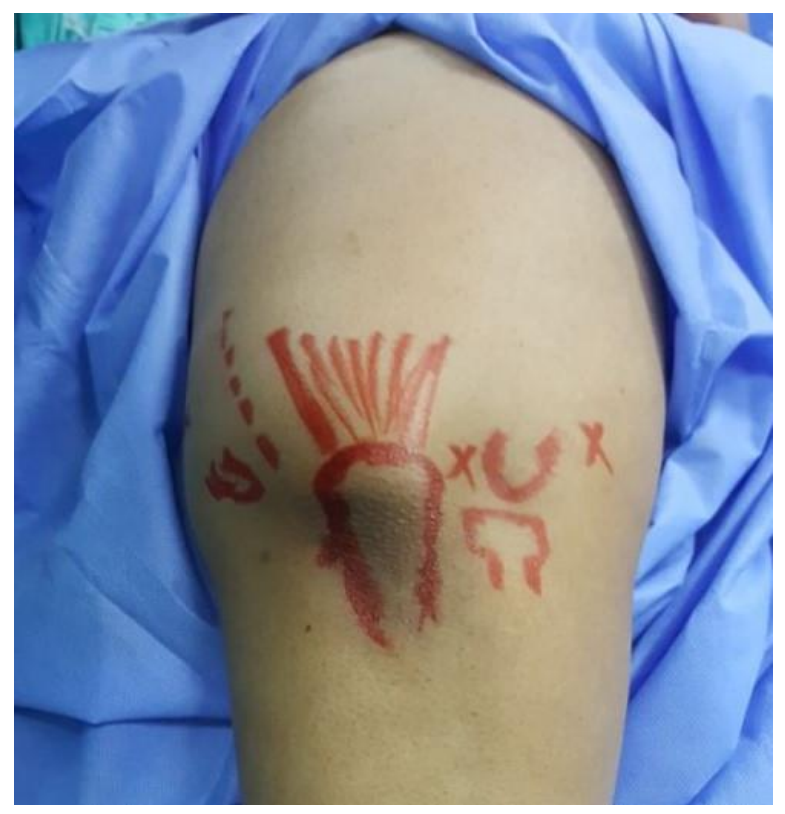

Figure (1): superficial skin landmarks.

An 18-gauge syringe needle was introduced into the lateral soft spot the joint was injected with approximately 20 to $30 \mathrm{~mL}$ of saline. Joint distention pushes the neurovascular structures more anterior, thereby protecting them from iatrogenic injury. The Free back-flow of fluid confirms correct intra-articular placement of the needle. The proximal anteromedial portal (viewing portal) was created approximately $2 \mathrm{~cm}$ proximal to the medial epicondyle and just anterior to the medial intermuscular septum. To protect against injury of the sensory nerves, the blunt trocar was inserted through the portal and aimed toward the center of the joint with maintaining contact with the anterior surface of the humerus. 


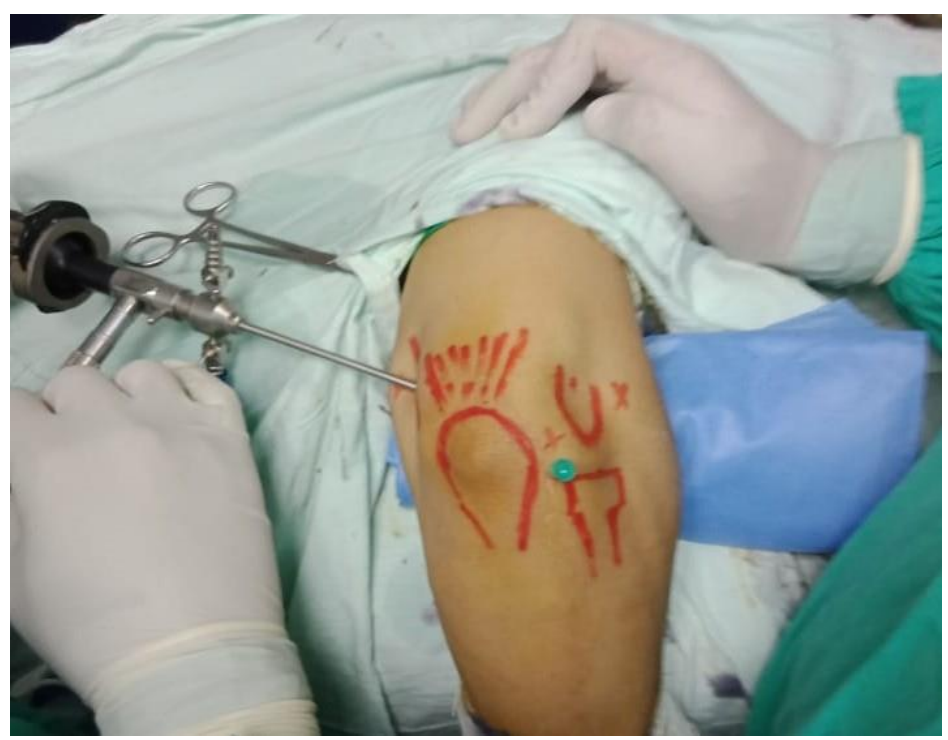

Figure (2): The proximal anteromedial portal (viewing portal) the lateral soft spot.

The back flow of fluid through the cannula confirms entry into the joint. The $4 \mathrm{~mm}, 30^{\circ}$ arthroscope was inserted into the joint. The lateral capsule and radio capitellar articulation were easily inspected. The joint capsule was examined for thickening, scarring and inflammation. Then the proximal anterolateral portal $(2 \mathrm{~cm}$ proximal and $1 \mathrm{~cm}$ anterior to the lateral epicondyle) was identified (working portal).

After a diagnostic arthroscopy, which allowed visualization of the entire anterior aspect of the elbow and perfect evaluation of the lateral structures, the area of damage to the ECRB was identified and resected off the anterior aspect of the lateral epicondyle. Once the portal was established, a small capsular window was made, a proximal anterolateral portal was made to allow a retractor to be placed to improve visualization and to protect the radial nerve. The shaver was then placed into the proximal anterolateral portal and portion of the lateral capsule was resected and revealed the underlying common extensor origin.

(a)

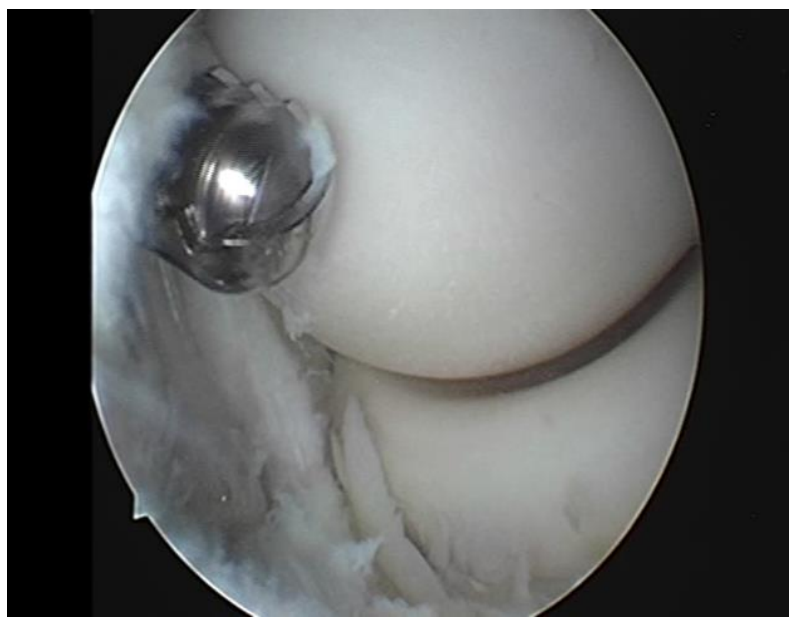

(b)

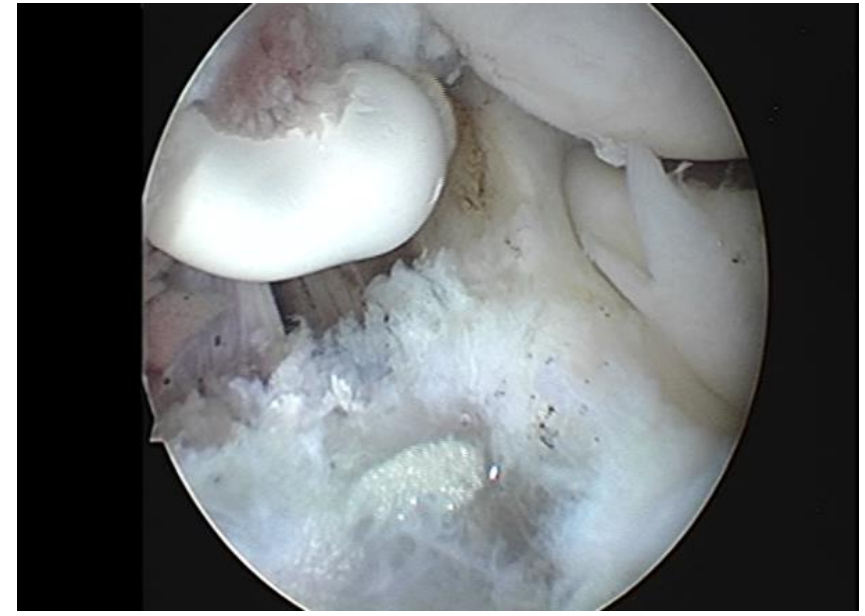

Figure (3): Arthroscopic appearance of ECRB tendon release by shaver (a), radiofrequancy device(b).

The shaver was exchanged for a monopolar radiofrequency device. The ECRB origin was then completely released from its insertion and the tendinosis tissue ablated until only healthy tendon remained or the fleshy ECRL tendon appeared. Once the entire pathologic tendon was removed, the anterior aspect of the lateral epicondyle was lightly decorticated in some cases. To protect the lateral ligamentous structures, care was taken not to extend the release posterior to a line bisecting the radial head and not to extend beyond radial head distally to avoid PIN injury. The portal sites were closed with simple sutures, and a sterile dressing was applied. 


\section{Post-operative Management:}

In the recovery area, once the patients had regained alertness and orientation, a neurovascular examination is done to ensure that no a neurovascular compromise had occurred.

The patients were followed up in outpatient clinics at Al-Azhar University Hospital two weeks postoperative then at six weeks and six months postoperatively. All previously mentioned scores were recorded at each time interval mentioned for follow up.

The study was approved by the Ethics Board of Al-Azhar University and an informed written consent was taken from each participant in the study.

\section{RESULTS}

Table (2) showed that the mean overall MEPI score in this study was improved from 71.33 points preoperative (range: 55 - 85 points) to 92.55 points (range: $70-100$ points) at six months post-operative.

The Nirschl staging score in our study improved from mean 4.13 points preoperatively (range: 1 - 7 points) to mean 1.73 points (range: $1-5$ points) at six months post-operative.

The DASH score quantifies pain and disability related to the upper extremity and ranges between 0 and 100 points. The lower DASH scores represent less pain and disability. Thus, in this study, a DASH score below 14 points was considered a good result. In our study, DASH score improved from a mean of 28.53 points preoperative to 3.60 points at six months post-operative. The mean VAS improved from mean 5.27 points preoperative (range: 3 - 8) to mean 1.47 points (range: 0 - 5) at six months post-operative. Two complications were reported: 2 patients had ulnar nerve injury. One (sensory and motor) still not recovered with hypothenar wasting and some trophic changes and undergone exploration in which only neurolysis has been done and the nerve was found intact and the other patient after one-month followup, sensory function was partially restored. In addition, at the fourth month follow-up, the patients' sensory, motor functions had fully recovered with just supportive medical treatment.

Table (2): comparison between pre, postoperative scores.

\begin{tabular}{|c||c|c|c|c|}
\hline Mean & $\begin{array}{c}\text { Pre- } \\
\text { Op. }\end{array}$ & $\begin{array}{l}\text { 2 weeks } \\
\text { post-op. }\end{array}$ & $\begin{array}{l}\text { 6 weeks } \\
\text { post-op. }\end{array}$ & $\begin{array}{c}6 \text { MO. } \\
\text { Post- } \\
\text { Op. }\end{array}$ \\
\hline \hline MEPI & 71.33 & 80.8 & 84.1 & 92.55 \\
\hline DASH & 28.53 & 15.1 & 10.3 & 3.6 \\
\hline Nirschl & 4.13 & 2.9 & 2.1 & 1.73 \\
\hline VAS & 5.27 & 2.4 & 2.0 & 1.47 \\
\hline
\end{tabular}

\section{DISCUSSION}

The mean overall MEPI in this study improved from 71.33 points pre-operative (range: $55-85$ points) to 92.55 points (range: $70-100$ points) at six months post-operative. Our results are consistent with that of Jerosch and Schunck ${ }^{(12)}$ who showed that mean MEPI posto-peratively were 93.6 points and 89 points respectively. Kim et al. ${ }^{(13)}$ carried out a study on 19 patients underwent arthroscopic simple release for recalcitrant lateral epicondylitis between 2004 and 2008, they noted that the MEPI improved from a median of 45 points to 95 points.

The Nirschl staging score in our study improved from mean 4.13 points pre-operative (range: $1-7$ points) to mean 1.73 points (range: $1-5$ points) at six months postoperative.

The DASH score quantifies pain and disability related to the upper extremity and ranges between 0 and 100 points. A study on a non-clinical population (14) indicated that the DASH score in healthy employed adults is $13 \pm 15$. In our stud, DASH score improved from a mean of 27.33 points pre-operative to 3.60 points at six months post-operative. Indeed this result was a somewhat good result in comparison to other studies. Wada et al. ${ }^{(15)}$ in their study showed that the mean postoperative DASH score was 10.6 points (range: 0-50). Othman ${ }^{(16)}$ in his study on 33 patients comparing the arthroscopic versus percutaneous release in treatment of chronic tennis elbow, noticed that the mean DASH score of the 14 patients who underwent arthroscopic release, improved from 72 points to 48 points post-operatively and he concluded that the arthroscopic treatment of lateral epicondylitis gives more favorable results in comparison to percutaneous one. This result is consistent with our result. Rhyou and Kim ${ }^{(17)}$ in their retrospective study on 39 patients who underwent arthroscopic treatment for refractory lateral epicondylitis from November 2003 to October 2009. They grouped 20 patients treated with arthroscopic release of the ECRB origin. They

noted that the mean DASH score in improved from 54.6 points to 6.1 points.

In the current study the mean VAS improved from mean 5.27 points pre-operative (range: $3-8$ ) to mean 1.47 points (range: 0 - 5) at six months postoperative. Owens et al. ${ }^{(18)}$ in their retrospective review of 16 patients with lateral epicondylitis treated with an arthroscopic release of the ECRB between January 1995 and November 1998 with a minimum of 1-year clinical follow-up. They noted that the mean post-operative VAS was 0.58 point (range: 0-3).

Szabo et al. ${ }^{(19)}$ in their study, which evaluated three surgical methods for treatment of lateral epicondylitis, found that the mean VAS in the 
arthroscopic group improved from 5.2 points to 1 point post-operatively.

Beker et al. ${ }^{(20)}$ in their study stated that the overall results of their clinical series were quite encouraging as most patients $(95 \%)$ reported that they were "much better" or "better" as a result of their arthroscopic release. Also Grewal et al. ${ }^{(21)}$ in their study upon 36 patients with chronic lateral epicondylitis underwent arthroscopic release reported that the average satisfaction was 8 over 10 . In comparison to the study by Owens $\boldsymbol{e t}$ al. ${ }^{(18)}$, who reported that; overall satisfaction was extremely positive in the 12 patients evaluated. Ten of the 12 patients $(83.3 \%)$ reported feeling much better because of their surgery, 2 reported feeling better, and none reported feeling the same or worse.

\section{CONCLUSION}

The elbow arthroscopy has a vital role in diagnosing and treating many elbow disorders. The complex anatomy in a relatively small space, proximity of the major nerves and vessels and lack of surgeon familiarity all contribute to the steep learning curve of elbow arthroscopic surgery.

Clinical outcomes of an arthroscopic release of the ECRB tendon showed to be a safe and effective and minimally invasive and reproducible procedure with marked post-operative increase in function within a short rehabilitation period. Arthroscopic release beside it effectively treats lateral epicondylitis, it also affords visualization of the joint space to the address associated intra-articular pathology. Pain relief and early restoration of elbow function can be reliably achieved with minimal morbidity and a high rate of patient satisfaction after the arthroscopic release of ECRB for lateral epicondylitis.

\section{REFERENCES}

1. Field LD, Savoie FH (1998): Common elbow injuries in sport. Sports Med., 26(3): 193-205.

2. Walrod BJ, Boucher LC, Conroy MJ et al. (2014): Lateral epicondylitis overview. http:// emedicine. medscape. com/ article/ 96969-overview.

3. Maffulli N, Khan KM, Puddu GC (1998): Overuse tendon conditions: time to change a confusing terminology. Arthroscopy, 14: 840-843.

4. Kraushaar BS and Nirschl RP (1999): Tendinosis of the elbow: clinical features, and findings of histological, immunohistochemical and electron microscopy studies. JBJS Am., 81: 259-278.

5. Khan KM, Cook JL, Maffulli $\mathbf{N}$ (2000): Tendinopathy in the active person: separating fact from fiction to improve clinical management. Am J Med Sports, 89: 840843.
6. Kraushaar BS and Nirschl RP (1999): Tendinosis of the elbow: clinical features, and findings of histological, immunohistochemical and electron microscopy studies. JBJS Am., 81:259-278.

7. Khan KM, Cook JL, Maffulli N (2000): Tendinopathy in the active person: separating fact from fiction to improve clinical management. Am J Med Sports, 89:43.

8. Maffillli N, Regine R, Carrillo F et al. (1990): Tenniselbow: an ultrasonographic study in tennis players. Br J Sports Med., 24:151-155.

9. Coonrad RW and Hooper WR (1973): Tennis elbow: its course, natural history, conservative and surgical management. J Bone Joint Surg., 55A: 1 177- 1182.

10. Terra BB, Rodrigues LM, Nahssen Filho A et al. (2015): Arthroscopic treatment for chronic lateral epicondylitis. Revista Brasileira de Ortopedia, 50(4):395402.

11. Babaqi AA, Kotb MM, Said HG et al. (2014): Shortterm evaluation of arthroscopic management of tennis elbow; including resection of radio-capitellar capsular complex. Journal of orthopaedics, 11(2):82-6.

12. Jerosch J and Schunck J (2006): Arthroscopic treatment of lateral epicondylitis: indication, technique and early results. Knee Surg Sports Traumatol Arthrosc., 14(4):379382.

13. Kim JW, Chun CH, Shim DM et al. (2011): Arthroscopic treatment of lateral epicondylitis: comparison of the outcome of ECRB release with and without decortication. Knee Surg Sports Traumatol Arthrosc., 19(7):1178-1183.

14. Jester A, Harth A, Germann G (2005): Measuring levels of upper extremity disability in employed adults using the DASH Questionnaire. J Hand Surg Am., 30(5):1074e1071-10e1010.

15. Wada T, Moriya T, Iba $K$ et al. (2009): Functional outcomes after arthroscopic treatment of lateral epicondylitis. J Orthop Sci., 14(2):167- 174.

16. Othman AM (2011): Arthroscopic versu percutaneous release of common extensor origin for treatment of chronic tennis elbow. Arch Orthop Trauma Surg., 131(3):383--388.

17. Rhyou IH and Kim KW (2013): Is posterior synovial plica excision necessary for refractory lateral epicondylitis of the elbow? Clin Orthop Relat Res., 471(1):284- 290.

18. Owens BD, Murphy KP, Kuklo TR (2001): Arthroscopic release for lateral epicondylitis. Arthroscopy, 17(6):582--- 587

19. Szabo SJ, Savoie FH, Field LD et al. (2006): Tendinosis of the extensor carpi radialis brevis: an evaluation of three methods of operative treatment. J Shoulder Elbow Surg., 15(6):721--- 727.

20. Baker CL and Baker CL (2013): Arthroscopic Treatment of Lateral Epicondylitis Operative Techniques in Orthopaedics, 23(2):91-97.

21. Grewal R, MacDermid JC, Shah P et al. (2009): Functional outcome of arthroscopic extensor carpi radialis brevis tendon release in chronic lateral epicondylitis. J Hand Surg Am., 34(5):849-857. 